Article

\title{
An SVM-Based Classifier for Estimating the State of Various Rotating Components in Agro-Industrial Machinery with a Vibration Signal Acquired from a Single Point on the Machine Chassis
}

\section{Ruben Ruiz-Gonzalez ${ }^{1, *}$, Jaime Gomez-Gil ${ }^{1}$, Francisco Javier Gomez-Gil ${ }^{2}$ and Víctor Martínez-Martínez ${ }^{1}$}

1 Department of Signal Theory, Communications and Telematics Engineering, University of Valladolid, Valladolid 47011, Spain; E-Mails: jgomez@tel.uva.es (J.G.-G.); vmarmar@ribera.tel.uva.es (V.M.-M.)

2 Department of Electromechanical Engineering, University of Burgos, Burgos 09006, Spain; E-Mail: fjggil@ubu.es

* Author to whom correspondence should be addressed; E-Mail: rruigon@ribera.tel.uva.es; Tel.: +34-6366-81022; Fax: +34-9834-23667.

External Editor: Gonzalo Pajares Martinsanz

Received: 9 September 2014; in revised form: 20 October 2014 / Accepted: 23 October 2014 / Published: 3 November 2014

\begin{abstract}
The goal of this article is to assess the feasibility of estimating the state of various rotating components in agro-industrial machinery by employing just one vibration signal acquired from a single point on the machine chassis. To do so, a Support Vector Machine (SVM)-based system is employed. Experimental tests evaluated this system by acquiring vibration data from a single point of an agricultural harvester, while varying several of its working conditions. The whole process included two major steps. Initially, the vibration data were preprocessed through twelve feature extraction algorithms, after which the Exhaustive Search method selected the most suitable features. Secondly, the SVM-based system accuracy was evaluated by using Leave-One-Out cross-validation, with the selected features as the input data. The results of this study provide evidence that (i) accurate estimation of the status of various rotating components in agro-industrial machinery is possible by processing the vibration signal acquired from a single point on the machine structure; (ii) the vibration signal can be acquired with a uniaxial accelerometer, the orientation of which does not significantly affect the classification
\end{abstract}


accuracy; and, (iii) when using an SVM classifier, an 85\% mean cross-validation accuracy can be reached, which only requires a maximum of seven features as its input, and no significant improvements are noted between the use of either nonlinear or linear kernels.

Keywords: Support Vector Machine (SVM); predictive maintenance (PdM); agricultural machinery; condition monitoring; fault diagnosis; vibration analysis; feature extraction and selection; pattern recognition

\section{Introduction}

Agro-industrial machinery has a high initial investment and requires regular maintenance if further expensive repairs are to be avoided [1]. The need for machine maintenance programs and their appropriateness is a well-argued topic in the industry, which is reflected in the literature [2]. Early detection of a mechanical component that is malfunctioning will lead to its prompt replacement, thereby avoiding more costly repairs in the future.

Nowadays, many predictive maintenance techniques are employed, in order to reduce hazards and subsequent failures of machinery [1-3]. According to Scheffer et al. [4], the main such techniques are vibration monitoring, acoustic emission, oil analysis, particle analysis, corrosion monitoring, thermography, and performance monitoring.

Vibration analysis is a non-intrusive method that is widely employed in machinery status inspections, mainly on rotating equipment including engines, turbines, and compressors, among others [4-6]. In the case of machinery with no vibration isolation, the vibration signal propagates throughout the whole structure of the machine with moderate attenuation. The propagation of these vibrations makes it possible to monitor certain rotating components by placing an accelerometer at a different point on the machine structure [7]. However, the propagation of vibrations has a disadvantage, in so far that it transmits various vibration signals from various other machine components, in addition to the signal of interest, making it more difficult to identify the relevant signal [8].

Vibration signals from rotating components are usually analyzed in the frequency domain, because significant peaks in the signal spectrum appear at frequencies that are related to the rotation frequency of the component [4]. Various authors have performed this analysis using fast Fourier transform [9], short-time Fourier transform [10], the wavelet transform [11-15], the S-transform [16], and the Hilbert-Huang transform [17-20], among others. Due to the relationship between the rotation frequency of the machine component and the highest peaks in the spectrum signal, experts can estimate the status of machine components by looking for patterns in the spectrum signal. Nevertheless, doing so requires expert analysis of the vibration signal spectrum, which implies detailed knowledge of the machine, the way it functions and full information on the rotation speed of the component. Automated systems have been proposed to estimate the status of machine components using frequency analysis in the absence of expert analysis [19,21]. These systems incorporate knowledge of the machine component to extract characteristics from the spectrum signal and to estimate its status on the basis of these characteristics. 
A Support Vector Machine (SVM) [22] is a supervised learning model widely used in the discipline of pattern recognition for classifying purposes. Due to its learning and generalization capabilities it is well suited for the implementation of estimation methods, which are widely required in automated diagnosis systems. According to the literature, many SVM-based applications have successfully been implemented [23], both for classification [24] and nonlinear regression [25]. Numerous improvements have been proposed over recent years that focus specifically on vibration monitoring in machinery fault diagnosis [26,27]. Widodo and Yang [28] offered a very thorough review of the latest major advances in the field of SVM-based vibration analysis for predictive maintenance.

Although a good deal of research has previously examined SVMs in machinery predictive maintenance $[21,28]$, to the best of our knowledge, automatic prediction of the state of various rotating components in an agro-industrial machine by employing only one vibration signal acquired from a single point on the machine chassis, has not been conducted in previous research.

The purpose of this article is to present evidence to assess the feasibility of estimating the state of various rotating components in agro-industrial machinery by employing one vibration signal acquired from a single point on the machine chassis as the system input. The following five rotating component states in an agricultural harvester were selected to assess that estimation capability: (1) engine speed status (high speed/low speed); (2) threshing cylinder operating status (on/off); (3) threshing cylinder balance status (balanced/unbalanced); (4) straw chopper operating status (on/off); and (5) straw chopper balance status (balanced/unbalanced).

\section{Background}

This section comprises some fundamentals about vibration analysis in agro-industrial machinery, classification in supervised machine learning, feature extraction and selection, and SVM-based classification.

\subsection{Vibrations in Agro-Industrial Machinery}

Vibration can be defined as the repeated motion of a certain component back and forth from a given position. Accelerometers are sensors that measure proper acceleration. These devices are the most widely used for capturing vibration signals in rotating machinery applications. Typical accelerometers capture signals in frequency ranges from $1 \mathrm{~Hz}$ to $10 \mathrm{kHz}$ [4].

The most common defects causing high vibration levels in machinery, in accordance with Scheffer et al. [4], are: unbalance of rotating parts, misalignment of couplings and bearings, bent shafts, worn or damaged gears and bearings, bad drive belts and chains, torque variations, electromagnetic forces, aerodynamic forces, hydraulic forces, looseness, rubbing, and resonance. When machinery rotating components operate at high speeds or under harsh operating conditions for a long time, some of these defects start to appear.

Vibrations can reveal the presence of machinery defects. Usually, vibrations on rotating components appear at specific frequencies, which are characteristic of each specific component and also depend on the component rotation speed and other properties [19,21]. Traditionally, depending on the vibration amplitude at those specific frequencies, the severity of the defects can be assessed. 
Therefore, plenty of information on the condition of a component, e.g., possible deterioration, can be detected by analyzing the vibration characteristics of isolated components [4].

In addition, the vibration signals of a specific machine component can be acquired from almost any point on the machine structure, even though the signals will be slightly attenuated, due to their propagation throughout the machine structure and the imperfect isolation of the main sources of vibration. Propagation complicates data processing and the extraction of useful information, because information from several machine components is mixed. It nevertheless greatly simplifies the data acquisition stage, as just a sensor may be installed at a single point on the machine.

\subsection{Classification in Supervised Machine Learning}

Machine learning, as a sub-field of artificial intelligence in computer science, deals with intelligent systems that can modify their behavior in accordance with the input data. Intelligent systems must have the capability of deducing the function that best fits the input data, in order to learn from the data. Machine learning can be divided into unsupervised and supervised learning, depending on the information that is available for the learning process. Unsupervised machine learning undertakes the inference process by using an unlabeled training set, i.e., without any information on the desired output, and it seeks to deduce relationships by looking for similarities in the dataset. Meanwhile, supervised machine learning assumes that a labeled training set, for which the desired output is completely known, is available.

Classification, as a branch of supervised learning, is defined as the process of identifying the class to which a previously unseen observation belongs, based on previous knowledge given by a training dataset that contains instances the category membership of which is certain. Any algorithm which performs classification tasks, i.e., the mapping of input data to an assigned class, is called a classifier.

Classifiers must be trained, based on previous knowledge, in order to function properly. The training process makes use of a sample of $N$ observations, the corresponding classes of which are certain. This sample of $N$ observations is typically divided into two subsamples: the training and the test datasets. Firstly, the training dataset is used in the process of computing a classifier that is well-adapted to these data. Then the test dataset is used to assess the generalization capability of the previously computed classifier.

Both the misclassification rate and the success rate in the test dataset are commonly used as quality measurements to assess classifier performance. The misclassification rate is defined as the proportion of observations which are wrongly assigned to an incorrect class. It is expressed as follows:

$$
M R=\frac{\text { Number of Incorrect Classifications }}{\text { Total Number of Classifications }}
$$

Alternatively, the success rate (also called the hit rate) is defined as the proportion of observations that are properly assigned to the corresponding class and is calculated as follows:

$$
S R=\frac{\text { Number of Correct Classifications }}{\text { Total Number of Classifications }}=1-M R
$$


The $k$-fold cross-validation is an enhanced method of evaluating classifier performance, especially with small training and test datasets. In this method, the original sample of $N$ observations is randomly partitioned into $k$ subsamples of equal size. From those $k$ subsamples, a single subsample is retained as the test dataset, and the remaining $k-1$ subsamples are used as the training dataset. The $k$-fold cross-validation repeats this training and test process $k$ times, using each of the $k$ subsamples only once as the test dataset. Cross-validation accuracy is calculated as the average of the success rate obtained for each of the $k$ different test datasets. When $k=N, k$-fold cross-validation is also known as leave-one-out cross-validation.

Many different classifiers have been proposed in the literature [29,30]. Some of the main ones include k-nearest neighbor classifier, Bayes classifier, logistic regression, Fisher's linear discriminant, decision tree, Artificial Neural Networks (ANN), and Support Vector Machines (SVM). An SVM classifier is used in this article and hence SVM is described in greater detail in Section 2.4.

\subsection{Feature Extraction and Selection for Classification}

Machine learning systems, including classifiers, are typically required to process large volumes of information. The application of dimensionality reduction techniques to the input data prevents the classifier from processing too much data and improves its performance. Dimensionality reduction, within statistical machine learning field, is defined as the process of reducing the number of variables of a dataset while retaining most of its degrees of freedom, thereby simplifying the subsequent classification problem. Feature extraction and selection are methods to accomplish dimensionality reduction.

Feature extraction [29] consists in reducing the dimensions of a $d$-dimensional input data vector by transforming it into a new $m$-dimensional output data vector, where $m<d$. The resulting $m$-dimensional vector, called feature vector, should retain from the original vector most of the useful information for the subsequent classification stage. This property is often referred as degrees of freedom preservation. Attending to their data type, features can be categorical, ordinal, integer-valued, or real-valued. A very wide variety of feature extraction algorithms have been proposed in the literature [31-34]. A taxonomy of these algorithms exists on the basis of their relationship to specific mathematical fields. The most popular such categories are nonlinear, statistical and transformed-domain based. Some of the nonlinear feature extraction algorithms are Correlation Dimension [35], Kolmogorov Complexity [36], Lempel-Ziv Complexity [31,37], Approximate Entropy [38], and Sample Entropy [39]. Classical time-domain based methods of statistical feature extraction include Mean Value, Standard Deviation, Skewness, Kurtosis, Average Power, and Shannon Entropy [40]. Some of the notable frequency domain feature extraction techniques are Spectral Entropy [32], Median Frequency [33,41], Bandwidth Containing 90\% of the Signal Energy, and Relative Wavelet Packet Energy [34].

Feature selection [29] involves choosing, among an original set of features of size $m$, the subset of size $n$ that best represents the original set and that yields the smallest classification error. The feature selection process can be conducted, among other methods by means of Exhaustive Search or Sequential Forward/Backward Floating Search [29]. On the one hand, Exhaustive Search explores all the possible subsets, i.e., $2^{m}$ if $n$ is a free parameter, or $\left(\begin{array}{c}m \\ n\end{array}\right)$ if $n$ is a preset constant. This method therefore guarantees the selection of the best subset, although its use of computational resources is 
excessive. On the other hand, Sequential Forward/Backward Floating Search restricts the search to a smaller subtree by only allowing feature deletion and addition at each step. Consequently, this method presents a more affordable computational load, but it fails to guarantee optimal subset selection, even though it has been proven to yield suboptimal results that are almost optimal.

The performance improvements offered by feature extraction and selection techniques are linked to: (i) dimension reduction that mitigates the 'curse of dimensionality' problem and therefore reduces the risk of over-fitting [29,42]; and (ii) simplification of the resulting classifier, which results in using less memory and fewer computational resources [29].

\subsection{Support Vector Machines for Classification}

Support Vector Machines (SVM) is a statistical supervised machine learning technique, used both for classification and for regression purposes. Originally proposed by Vapnik and Cortes [22,43], in 1995, although its principles and derivation differ from those of Artificial Neural Networks (ANN), some authors sometimes consider SVMs as a special kind of ANN [44]. However, many authors refuse to do so, due to essential differences between SVM and ANN techniques [45]. While SVM mechanisms are mainly based on a rigorous geometrical and statistical approach, ANNs try to emulate the behavior of the human brain and its neural system.

The original SVM proposal was aimed at both the binary classification problem, considering only two possible classification classes, and the multiclass classification problem, which considers more than two classification classes.

Binary linear SVM classification performs the calculation of the optimal hyperplane decision boundary, separating one class from the other, on the basis of a training dataset. Optimality can be understood, depending on whether perfect classification of the training dataset is feasible and desired, in two separate ways:

- If perfect separability of training dataset classes can be achieved, a Hard Margin optimality can be used. In this case, the hyperplane decision boundary is chosen to maximize the distance from the hyperplane to the nearest training data point.

- If perfect classification is not desired or if it is impossible, a Soft Margin optimality is used. In this case, the hyperplane selection is a customizable tradeoff between minimizing the misclassification rate and maximizing the distance to the nearest properly classified training point.

The decision boundary hyperplane in SVM classification is calculated by employing the training dataset. This decision boundary is completely determined by the so-called Support Vectors, a subset of training input vectors which by themselves alone lead to the same decision boundary. After this hyperplane is determined, the SVM classifier is ready to be used with a different dataset from the one used in the training stage. The assigned class, labeled either +1 or -1 , depends on the side of the decision boundary on which the input vector falls. Figure 1 represents a graphical example of linear SVM-based classification, both in the case of linearly separable classes and non-linearly separable classes.

SVM multiclass classification usually tackles the classification and computation of the decision boundary by reducing the problem to a set of binary classification problems. The main such approaches are 
pairwise and one-versus-all classification methods [46]. Compact multiclass reformulations of the binary classification problem have also been proposed [46].

Figure 1. Representation of a Support Vector Machine (SVM)) classifier corresponding to (a) a linearly separable pattern, where the hyperplane totally separates green circles from red squares; and (b) a non-linearly separable pattern, where no hyperplane separates all the green circles from the red squares.

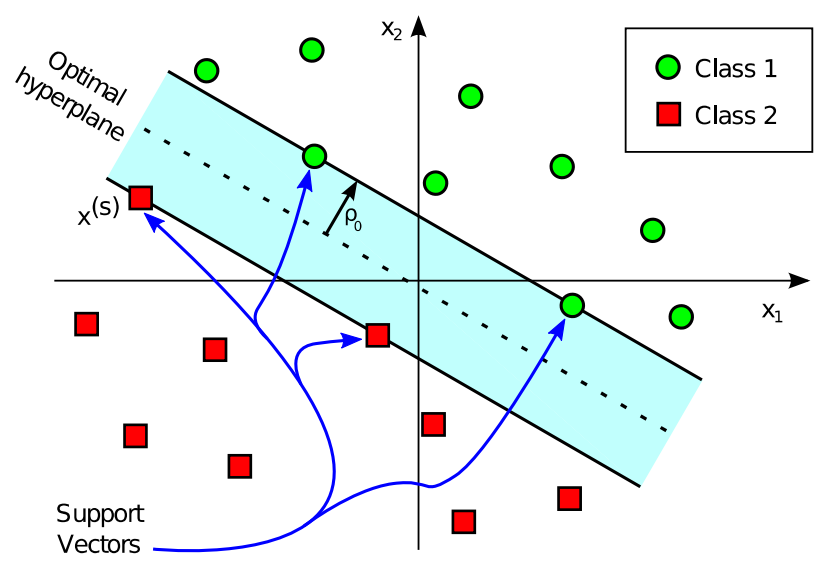

(a)

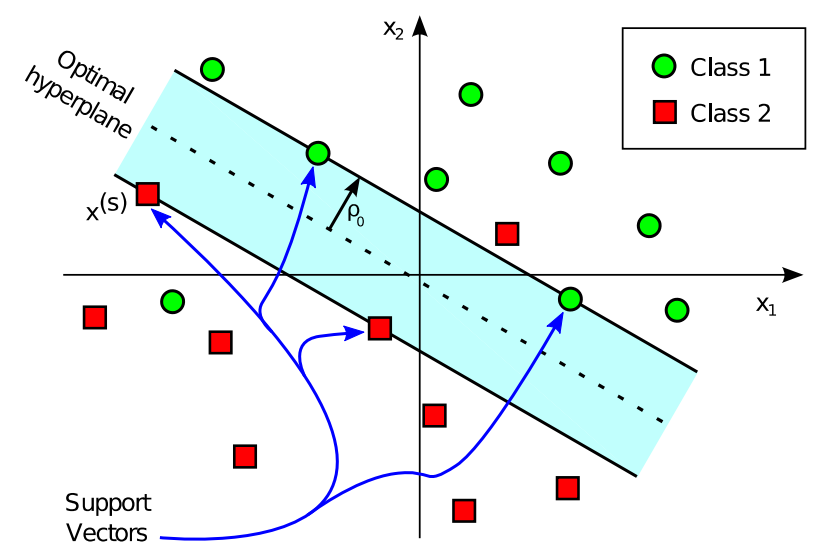

(b)

To be mathematically rigorous, the most general SVM linear binary classification problem can be stated as follows:

"Given a training dataset, $\left\{\boldsymbol{x}_{i}, d_{i}\right\}_{i=1}^{N}$, the goal is to compute the optimal weight vector $\boldsymbol{w}$, bias $b$, and slack variables $\xi$, such that satisfy the following constraints:

$$
\begin{gathered}
d_{i}\left(\boldsymbol{w}^{T} \boldsymbol{x}_{i}+b\right) \geq 1-\xi_{i}, \forall i=1,2, \ldots, N \\
\xi_{i} \geq 0, \forall i=1,2, \ldots, N
\end{gathered}
$$

and such that the following cost function is minimized:

$$
\Phi(\boldsymbol{w}, \xi)=\frac{1}{2} \boldsymbol{w}^{T} \boldsymbol{w}+C \sum_{i=1}^{N} \xi_{i}
$$

where, $\boldsymbol{x}_{i} \in \mathbb{R}^{m_{0}}$ denotes the $i$-th input vector, $d_{i} \in\{-1,1\}$ denotes the class corresponding to the $i$-th input vector, $\xi=\left\{\xi_{i}\right\}_{i=1}^{N}$ represents the slack variables, and the constant $C$ is a user-specified parameter that determines the tradeoff between misclassification and maximum inter-class margin."

In practice, most classification problems cannot be solved by using a simple hyperplane as the decision boundary. In such cases a more complex and elaborate decision boundary is required. SVM achieves this goal by increasing the dimensionality of the input space, of dimension $m_{0}$, by applying a nonlinear transformation, denoted by $\boldsymbol{\varphi}(\cdot)$, into a feature space of dimension $m_{f}>m_{0}$ (Figure 2). This transformation, $\varphi(\cdot)$, serves to reduce the misclassification probability in the transformed feature space. The most typical transformation functions, as in the case of ANNs, are radial basis functions, higher-order polynomials, and sigmoids. Figure 2 represents a graphical example of an SVM nonlinear classification. 
Figure 2. Representation of a Support Vector Machine classifier with a nonlinear kernel. Function $\varphi(\cdot)$ is the nonlinear transformation mapping vectors from (a) the input space to (b) the feature space.

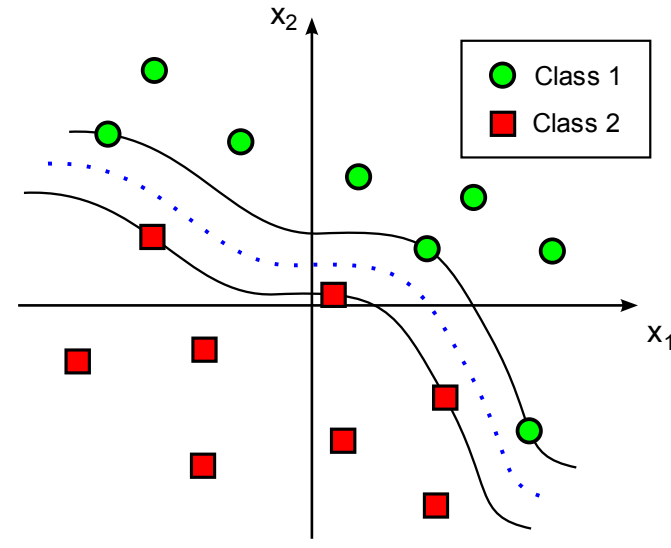

(a)

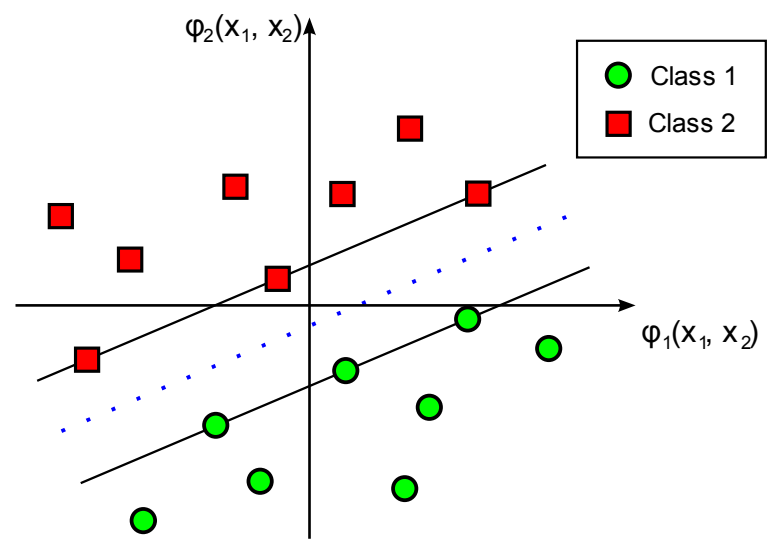

(b)

The boundary in the nonlinear classification problem is still a hyperplane, not in the original input space but in the feature space, and can be expressed as the points $\boldsymbol{\varphi}(\boldsymbol{x})$ that satisfy that:

$$
\boldsymbol{w}^{T} \boldsymbol{\varphi}(\boldsymbol{x})+b=0
$$

where, $\boldsymbol{x} \in \mathbb{R}^{m_{0}}$ and $\boldsymbol{\varphi}(\boldsymbol{x}) \in \mathbb{R}^{m_{f}}$.

Following the application of the Lagrange multipliers method, it has been shown that the optimal weight vector can be expressed as [44]:

$$
\boldsymbol{w}=\sum_{i=1}^{N} \alpha_{i} d_{i} \boldsymbol{\varphi}\left(\boldsymbol{x}_{i}\right)
$$

where, $\alpha_{i}$ stands for the Lagrange multiplier coefficients.

Therefore, the optimal decision boundary can be rewritten as:

$$
\sum_{i=1}^{N} \alpha_{i} d_{i} \boldsymbol{\varphi}\left(\boldsymbol{x}_{i}\right)^{T} \boldsymbol{\varphi}(\boldsymbol{x})+b=0
$$

Renaming $\quad u_{i}=\alpha_{i} d_{i} \quad$ and $\quad K\left(\boldsymbol{x}_{\boldsymbol{i}}, \boldsymbol{x}\right)=\boldsymbol{\varphi}\left(\boldsymbol{x}_{i}\right)^{T} \boldsymbol{\varphi}(\boldsymbol{x})=\boldsymbol{\varphi}(\boldsymbol{x})^{T} \boldsymbol{\varphi}\left(\boldsymbol{x}_{i}\right)=K\left(\boldsymbol{x}, \boldsymbol{x}_{\boldsymbol{i}}\right), \quad$ the decision function, $y$, can be expressed as:

$$
y=\sum_{i=1}^{N} u_{i} K\left(\boldsymbol{x}, \boldsymbol{x}_{\boldsymbol{i}}\right)+b
$$

In case of linear classifiers, $K\left(\boldsymbol{x}, \boldsymbol{x}_{\boldsymbol{i}}\right)$ is the conventional Euclidean inner product of the input vector $\boldsymbol{x}$ with the Support Vector $\boldsymbol{x}_{\boldsymbol{i}}$. In case of nonlinear classifiers, $K\left(\boldsymbol{x}, \boldsymbol{x}_{\boldsymbol{i}}\right)$ is the conventional Euclidean inner product of the nonlinear transformation $\boldsymbol{\varphi}(\boldsymbol{x})$ of the input vector $\boldsymbol{x}$ with the nonlinear transformation $\boldsymbol{\varphi}\left(\boldsymbol{x}_{\boldsymbol{i}}\right)$ of the Support Vector $\boldsymbol{x}_{\boldsymbol{i}}$.

The decision function in Equation (4) results in the architecture depicted in Figure 3, once the proper weights and Support Vectors have been computed in the training stage. Only the Support Vectors have to be considered, as they are the only vectors that generate non-zero $\alpha_{i}$ coefficients [44]. 
Classification is therefore performed by identifying the sign of the output value, $y$, in Equation (4). If $\operatorname{sign}(y)=+1$, then this input is labeled as class +1 and if otherwise as class -1 .

Figure 3. Architecture of a Support Vector Machine classifier. Inner product kernels, $K(\cdot, \cdot)$, denote the $m_{0}$-dimensional kernel inner product of the input vector with each of the $N_{s}$ Support Vectors.

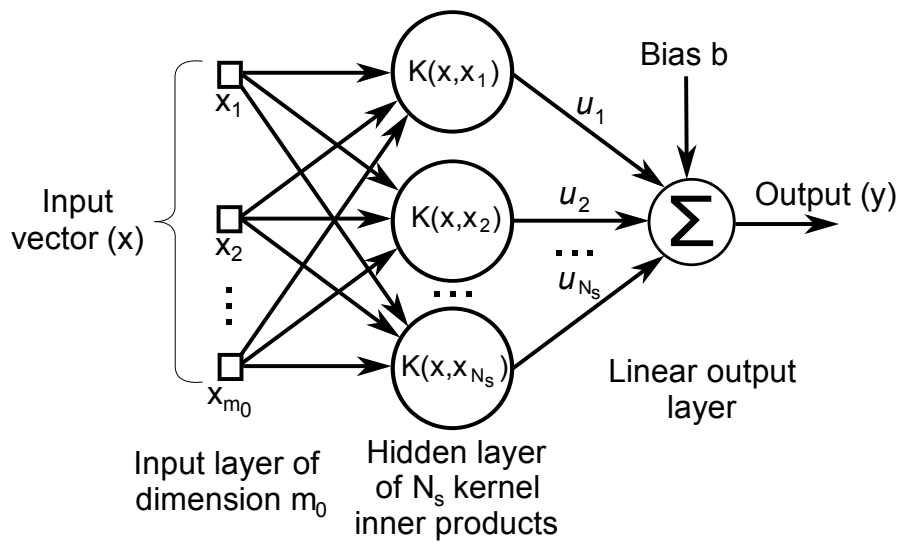

The most well-known and widely used nonlinear kernels are radial basis functions $(R B F)$, sigmoids, and polynomials. The $R B F$ kernel can be expressed as $K(\boldsymbol{x}, \boldsymbol{y})=\exp \left(-\gamma\|\boldsymbol{x}-\boldsymbol{y}\|^{2}\right)$, where $\gamma$ is a user-defined parameter; the sigmoidal kernel can be expressed as $K(\boldsymbol{x}, \boldsymbol{y})=\tanh \left(\gamma \boldsymbol{x}^{\mathbf{T}} \boldsymbol{y}+c_{0}\right)$, where $\gamma>0$ and $c_{0}<0$ are user-defined parameters; and, the $d$-order polynomial kernel can be expressed as $K(\boldsymbol{x}, \boldsymbol{y})=\left(\gamma \boldsymbol{x}^{\mathbf{T}} \boldsymbol{y}+c_{0}\right)^{d}$, where $\gamma$ and $c_{0}$ are user-defined parameters and where $d$ denotes the polynomial degree. Other kernels may also be found, in addition to those listed above.

The underlying SVM training process undertakes the problem of minimizing a quadratic functional subject to linear constraints. This problem, known as Quadratic Programming, has a closed solution. Although the solution can be analytically computed by applying the Lagrange multipliers method, other computational methods are typically used, especially when the dimensionality of the problem becomes high. Some of these methods include, among others, Interior Point methods [47], the Sequential Minimal Optimization (SMO) algorithm [48,49], Incremental methods [50], and the Kernel-Adatron (KA) algorithm [51]. More information about the SVM training process has been gathered by Campbell and Ying [52].

Those readers eager to discover the rigorous mathematical statement and solution of the problem underlying Support Vector Machines are encouraged to read the comprehensive introduction to SVM provided by Haykin [44] or the in-depth work by Steinwart and Christmann [53].

\section{Materials and Methods}

The main processing stages performed in this study can be conceptualized as follows: (i) the data acquisition stage (Section 3.1); (ii) the preprocessing stage (Section 3.2); (iii) the feature extraction and selection stage (Section 3.3); (iv) the SVM-based classification stage (Section 3.4); and (v) the evaluation stage (Section 3.5). Figure 4 summarizes the main processing stages and contains a high-level description of the methods, which are explained in greater detail in the remainder of this section. 
Figure 4. Overall block diagram summarizing the main processing stages.
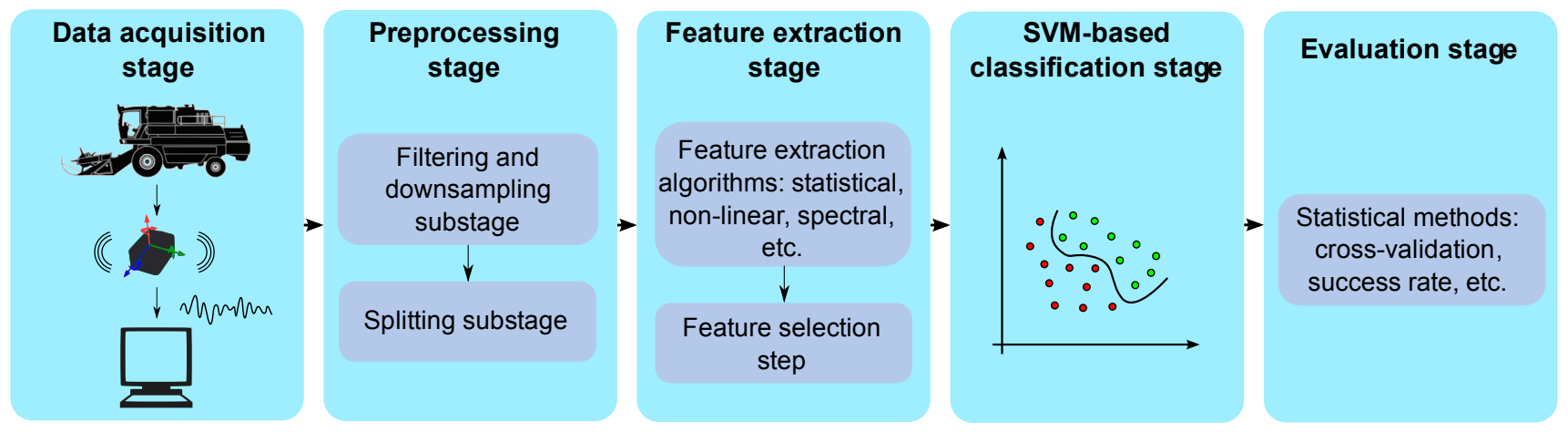

\subsection{Data Acquisition Stage}

Vibration data were experimentally obtained from an eleven-year-old New Holland TC56 harvester that had clocked 3800 working hours. Vibration signals were acquired from a stationary harvester operating in threshing mode. A Kistler 8690C50 triaxial accelerometer was used to measure the vibration signals on transverse, longitudinal and vertical axes (Figure 5). After several trial and error tests, the accelerometer sensor was placed on the left hand side of the harvester chassis, neither very close nor very far away from the rotating components under analysis (Figure 5). The sensor was positioned by using an adhesive mounting following the guidelines in Scheffer et al. [4]. This mounting method was selected because the frequency analysis in this article was bandlimited below $200 \mathrm{~Hz}$ and it permits accurate measurements within this frequency range [4]. Vibration signals were acquired using the NI Sound and Vibration Assistant software and a National Instruments (NI) data acquisition (DAQ) system. The data acquisition system was composed of an NI 9234 data acquisition module for analog input signals and an NI compact DAQ chassis NI $c D A Q-9172$, to connect the DAQ module to a laptop.

Figure 5. (a) Harvester schematic in which the red symbol represents the precise location of the accelerometer sensor on the chassis, the yellow cross represents the location of the engine, the blue cross represents the location of the threshing cylinder, and the orange cross represents the location of the straw chopper; (b) The coordinate axes of the accelerometer in this study were as follows: the $x$ axis was transverse to the front direction of the harvester, the $y$ axis pointed to the reverse direction of the harvester, and the $z$ axis was upward vertical with respect to the ground; (c) The experimental setup for data acquisition and a close up of the position of the Kistler 8690C50 triaxial accelerometer.

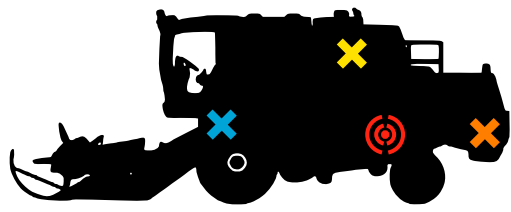

(a)

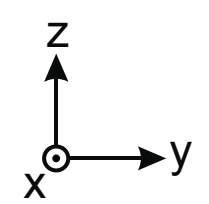

(b)

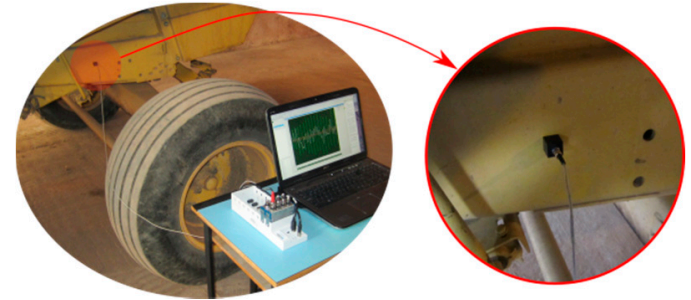

(c) 
A total of 18 different data acquisition processes were performed to acquire data on all the combinations of the following harvester working conditions: (i) engine speed status (high speed/low speed); (ii) threshing cylinder operating status (on/off); (iii) threshing cylinder balance status (balanced/unbalanced) in the on operating status; (iv) straw chopper operating status (on/off); and (v) straw chopper balance status (balanced/unbalanced) in the on operating status. The straw chopper was unbalanced on purpose by the breakage of a blade. The unbalance was provoked in this way, because blade breakage against stones is a frequent cause of unbalances. The threshing cylinder was unbalanced by adding an eccentric weight to it. The unbalance was provoked in this way, because the threshing cylinder can typically become unbalanced when its bars suffer from non-uniform wear, due to usage and an eccentric weight simulates the same effect.

Sixty-second long epochs or frames of machine operation were recorded for each of the 18 acquisition processes, using a sampling frequency of $1706.48 \mathrm{~Hz}$, which generated a total of 99,120 samples per epoch.

\subsection{Preprocessing Stage}

The acquired acceleration time-series data were first preprocessed in order to adapt them to the subsequent feature extraction stage. The entire preprocessing stage was divided into the following three sub-stages (Figure 6): ( ) a low-pass filtering sub-stage; (ii) a downsampling sub-stage; and (iii) a splitting sub-stage.

Figure 6. Block diagram representing the three preprocessing sub-stages.

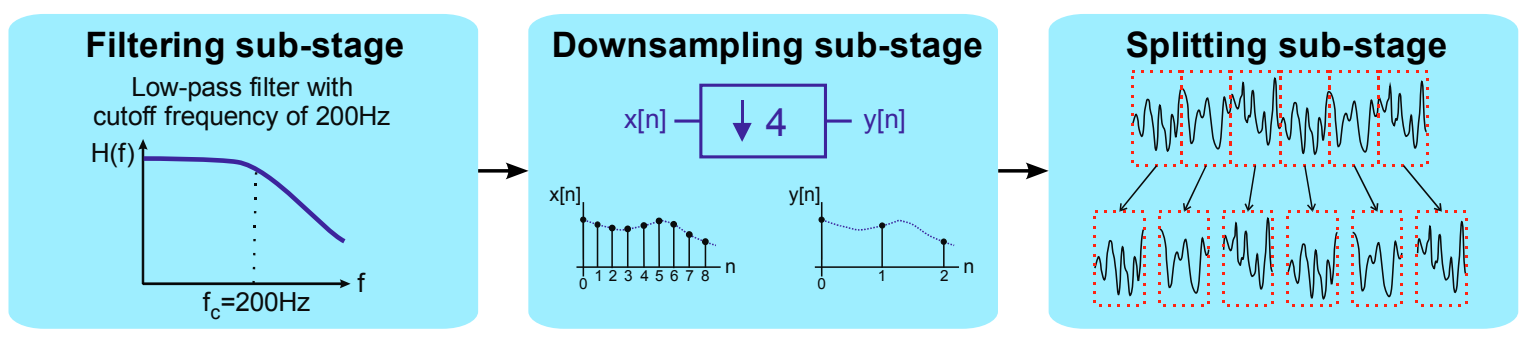

In the first sub-stage, low-pass filtering took place. A digital IIR elliptic low-pass filter, with a cutoff frequency of $200 \mathrm{~Hz}$, was applied to the input signal. The vibration frequencies of interest, which are the main harmonics of the components rotation speeds, are located within the range from 0 to $200 \mathrm{~Hz}$. This filtering was performed in order to remove noise and unwanted interferences to achieve a better performance.

Next, after filtering, the downsampling sub-stage took place. The input signal was decimated, in order to reduce the sampling frequency by a factor of $N_{f s}$, where $N_{f s} \in \mathbb{N}$ is the decimation ratio. In this article, a value of $N_{f s}=4$ was chosen, taking into account the frequency range of interest. Therefore, the effective sampling frequency was reduced after downsampling from $1706.5 \mathrm{~Hz}$, the one originally employed in the acquisition stage, to $426.625 \mathrm{~Hz}$.

Finally, the splitting sub-stage was conducted. The downsampled signal, coming from the second sub-stage, was then split into six epochs of about ten seconds, which was the frame size considered sufficient for keeping meaningful information on the vibration signal for the posterior feature 
extraction steps and for ensuring good frequency resolution in the subsequent FFT analysis. In this way, a total of 4130 samples per epoch were obtained.

All these preprocessing tasks were performed with $M A T L A B^{\circledR}$ program.

\subsection{Feature Extraction and Selection Stage}

This stage involves the dimensionality reduction of the input data-series coming from the previous stage. It is divided into two sub-stages: feature extraction and feature selection.

Firstly, the preprocessed data from the previous stage were brought in the feature extraction sub-stage, in order to achieve a simpler classifier. Then, the input signal for this stage, denoted as $x[n]$, where $n=1,2, \ldots, N=4130$, was used to extract the following features:

(1) Average Power $(P)$, defined as $\bar{P}=\frac{1}{N} \sum_{n=1}^{N} x[n]^{2}$. This feature quantifies the overall vibration intensity.

(2) Sample Entropy (SampEn), computed using the definition provided by Richman et al. [39]. This feature is a measurement of signal regularity that assigns higher values to more random data; for instance when multiple vibration sources are superposed.

(3) Spectral Entropy (SpecEn), computed in the same way as by Hornero et al. [33]. This feature was employed because of its capability to quantify the flatness of the spectrum. The more frequency peaks the signal has, the greater this feature becomes.

(4) Mean Value $(\bar{x})$, calculated as $\bar{x}=\frac{1}{N} \sum_{n=1}^{N} x[n]$. It reflects the amplitude of low frequency background vibrations.

(5) Median frequency $(M F)$, computed as the frequency which divides the power spectrum into two halves, each of which contains the same energy. It was calculated in the same way as by Hornero et al. [33].

(6) Standard Deviation ( $\sigma$ ), calculated by using the mean value $\bar{x}$ that has previously been defined, as the square root of the unbiased estimator of the variance, i.e., $\sigma=\sqrt{\frac{1}{N-1} \sum_{n=1}^{N}(x[n]-\bar{x})^{2}}$. This feature provides information on the width of the amplitude histogram distribution, supplying additional information on the shape of the vibration signal.

(7) Skewness $\left(s_{0}\right)$, calculated as the unbiased estimator $s_{0}=\frac{\sqrt{N(N-1)}}{N-2} \frac{\frac{1}{N} \sum_{n=1}^{N}(x[n]-\bar{x})^{3}}{\left(\sqrt{\frac{1}{N} \sum_{n=1}^{N}(x[n]-\bar{x})^{2}}\right)^{3}}$.

Skewness, which is a measure of histogram distribution asymmetry around its mean, can reflect vibration asymmetries due to mechanical faults.

(8) Kurtosis ( $\left.k_{0}\right)$, calculated as the unbiased estimator $k_{0}=\frac{N-1}{(N-2)(N-3)}\left((N+1) k_{1}-\right.$ $3(N-1))+3$, where $k_{1}=\frac{\frac{1}{N} \sum_{n=1}^{N}(x[n]-\bar{x})^{4}}{\left(\frac{1}{N} \sum_{n=1}^{N}(x[n]-\bar{x})^{2}\right)^{2}}$. This feature reflects the peakedness of the histogram, giving information on the distribution of the vibrations amplitude.

(9) Central Tendency Measurement (CTM). In the first place, the first-order differences scatter plot is constructed, representing $x[n+1]-x[n]$ on the $X$ axis against $x[n+2]-x[n+1]$ on the $Y$ axis. The proportion of points lying inside a circle of a certain fixed radius is then returned as a measurement of signal regularity. A radius of $0.05 \mathrm{~g}$ was selected in this study, 
which is an appropriate one for distinguishing the related classification classes. This feature offers a measurement of the randomness of the vibration signal, where a low value of this feature implies sharp changes in the vibration signal. Sharp changes in vibration signals may be caused both by high frequency vibrations or sudden transitions due to mechanical faults.

(10) Correlation coefficient (r) from the first-order differences scatter plot. As with the previous feature, the first-order differences scatter plot is constructed first, obtaining both $X[n]$ and $Y[n]$ vectors. Then the Pearson's linear correlation coefficient between both vectors is computed as: $r=\frac{\sum_{n=1}^{N-2}(X[n]-\bar{X})(Y[n]-\bar{Y})}{\sqrt{\sum_{n=1}^{N-2}(X[n]-\bar{X})^{2}} \sqrt{\sum_{n=1}^{N-2}(Y[n]-\bar{Y})^{2}}}$, where $\bar{X}$ and $\bar{Y}$ are the mean values of $X[n]$ and $Y[n]$, respectively. This feature offers a measurement of the unpredictability of the signal from the previous data; the higher the measure of $|r|$ the more predictable the signal is.

(11) Lempel-Ziv Complexity (LZC), computed as by Hornero et al. [33]. This feature offers a notion of complexity in a statistical sense. It characterizes the average information quantity within a signal and can therefore reflect the superposition of several vibration sources.

(12) Crest Factor $(C)$, calculated as $C=\frac{\max _{n}|x[n]|}{\sqrt{\frac{1}{N} \sum_{i} x[n]^{2}}}$, where $N$ is the number of samples of the time-series $x[n]$. This feature reflects the spikiness of the signal with respect to its RMS value and is therefore useful to assess the presence of mechanical faults.

All of the above algorithms were selected on the basis of the previous literature on vibration analysis [21,54-56] and by extrapolating ideas from studies in other fields [33,37,39].

Secondly, after having extracted these features from the preprocessed data-series, the most suitable features from among them all were selected in the feature selection sub-stage. The feature selection process was undertaken by using the Exhaustive Search method, which explores all of the possible feature subsets. With each of the explored subsets, linear SVM leave-one-out cross-validation was performed to assess the goodness of this subset. The feature subset with highest cross-validation accuracy was selected. The value of parameter $C$, involved in the SVM classification problem, was prefixed at 1 in all cases. Cross-validation accuracy was calculated for each classifier undertaking each of the five classification problems, corresponding to the five rotating component states of the agricultural harvester under consideration: (1) engine speed status (high speed/low speed); (2) threshing cylinder operating status (on/off); (3) threshing cylinder balance status (balanced/unbalanced); (4) straw chopper operating status (on/off), and (5) straw chopper balance status (balanced/unbalanced).

The choice of the Exhaustive Search method was possible due to the relatively small number of twelve features that were involved, as mentioned above. If more features were to be explored, it would be advisable to use Sequential Forward/Backward Floating Search for computational efficiency [29].

All the tasks of this stage were performed in the $M A T L A B^{\circledR}$ programming environment using the LIBSVM library [57,58].

\subsection{SVM-Based Classification Stage}

The classification stage took place once the previous processing stages had been performed. Among the huge variety of classifiers available, SVM classification was selected in this work because 
of its: (i) great generalization ability; (ii) low overtraining risk due to small datasets; and (iii) low computational load.

A different SVM-based classifier was employed for each of the five related classification problems, corresponding to the following five rotating component states of the agricultural harvester: (1) engine speed status (high speed/low speed); (2) threshing cylinder operating status (on/off); (3) threshing cylinder balance status (balanced/unbalanced); (4) straw chopper operating status (on/off); and (5) straw chopper balance status (balanced/unbalanced). The input of each classifier was the subset of features that led to maximum cross-validation accuracy (Section 3.3). If more than one subset led to the maximum value, only one of them was selected for the sake of simplicity. Each classifier provided one of the two classes associated with the input feature vector as its output.

For each of the five classifiers, the linear kernel and the radial basis function (RBF), the sigmoidal, and the third-order polynomial nonlinear kernels were employed, providing a comparison between their accuracy. These SVM kernels were selected, because they are the most typical and widely used. The $C$ parameter, involved in the SVM classification formulation, and the $\gamma$ and $c_{0}$ parameters, involved in the kernel, were optimized by conducting an exponential grid-search on these parameters [59]. The parameters that led to the highest cross-validation accuracy were selected.

The LIBSVM toolbox [57,58], running in the MATLAB ${ }^{\circledR}$ programming environment, was once again employed for classification tasks.

\subsection{Classifier Performance Evaluation Stage}

The leave-one-out cross-validation accuracy (Section 2.2), for each of the five individual classification problems under consideration, was computed to assess the goodness of the proposed classifying system. These five cross-validation accuracies, as well as the overall mean cross-validation accuracy, were used as a measurement of the accuracy of the SVM-based estimation method for each of the five aforementioned harvester states.

\section{Results}

The experimental results of the feature selection and classifier performance evaluation stages are presented in this section.

\subsection{Feature Selection}

The selection of the best features, following the methods explained in Section 3.3, led to the best cross-validation accuracies and best particular chosen features shown in Table 1. It can be appreciated that, in all cases, the required number of features is lower than or equal to seven and that the mean cross-validation accuracy is above $85 \%$, for all the three axes of the accelerometer. The best cross-validation accuracies and the number of features needed to achieve these are depicted in Figure 7. 
Table 1. Feature selection results for each of the three axes acquired by the triaxial accelerometer. The first row (number of features) shows the optimal number required to achieve the best cross-validation accuracy. The second row (best feature subset) shows all of the concrete feature subsets, giving the highest cross-validation accuracy as a list of numbers the legend of which corresponds to the list provided in Section 3.3. The subset employed for the subsequent classifier performance evaluation stage appears in bold. Each column corresponds to each of the rotating component classification problem under consideration.

\begin{tabular}{|c|c|c|c|c|c|c|}
\hline & \multicolumn{5}{|c|}{ Classification Problem } \\
\hline & & Engine Speed & $\begin{array}{l}\text { Threshing } \\
\text { Cylinder } \\
\text { Operation }\end{array}$ & $\begin{array}{c}\text { Threshing Cylinder } \\
\text { Balance }\end{array}$ & $\begin{array}{c}\text { Straw } \\
\text { Chopper } \\
\text { Operation }\end{array}$ & $\begin{array}{c}\text { Straw } \\
\text { Chopper } \\
\text { Balance }\end{array}$ \\
\hline \multirow{4}{*}{$\begin{array}{l}\text { Transverse } X \\
\text { axis of the } \\
\text { accelerometer }\end{array}$} & Number of features & 1 & 2 & 5 & 2 & 1 \\
\hline & $\begin{array}{c}\text { Best feature } \\
\text { subset(s) }\end{array}$ & $\{9\}$ & $\{10,11\}$ & $\{4,6,7,9,11\}$ & $\{2,8\} ;\{2,5\}$ & 5 \\
\hline & $\begin{array}{c}\text { Cross-validation } \\
\text { accuracy }\end{array}$ & $100 \%$ & $97.87 \%$ & $68.29 \%$ & $80.85 \%$ & $80 \%$ \\
\hline & Mean CVA & \multicolumn{5}{|c|}{$85.40 \%$} \\
\hline \multirow{4}{*}{$\begin{array}{l}\text { Longitudinal } \\
Y \text { axis of the } \\
\text { accelerometer }\end{array}$} & Number of features & 3 & 1 & 7 & 2 & 5 \\
\hline & $\begin{array}{l}\text { Best feature } \\
\text { subset(s) }\end{array}$ & $\begin{array}{l}\{\mathbf{6 , 7 , 1 0}\} \\
\{5,7,9\} \\
\{5,6,10\} \\
\{1,6,10\}\end{array}$ & $\{5\}$ & $\begin{array}{c}\{\mathbf{1}, \mathbf{2}, \mathbf{7}, \mathbf{8}, \mathbf{9}, \mathbf{1 1}, \mathbf{1 2}\} ; \\
\{1,2,4,7,8,11,12\} ; \\
\{1,2,4,6,7,11,12\} ; \\
\{1,2,3,5,7,8,11\}\end{array}$ & $\{4,11\}$ & $\{1,3,5,7,12\}$ \\
\hline & $\begin{array}{c}\text { Cross-validation } \\
\text { accuracy }\end{array}$ & $100 \%$ & $97.87 \%$ & $87.49 \%$ & $91.49 \%$ & $90 \%$ \\
\hline & Mean CVA & \multicolumn{5}{|c|}{$93.37 \%$} \\
\hline \multirow{4}{*}{$\begin{array}{l}\text { Vertical } \mathrm{Z} \text { axis } \\
\text { of the } \\
\text { accelerometer }\end{array}$} & Number of features & 1 & 2 & 6 & 6 & 4 \\
\hline & $\begin{array}{l}\text { Best feature } \\
\text { subset(s) }\end{array}$ & $\{9\}$ & $\{\mathbf{2}, \mathbf{1 1}\} ;\{2,3\}$ & $\{2,3,5,6,8,11\}$ & $\{1,4,5,7,8,10\}$ & $\{1,2,7,10\}$ \\
\hline & $\begin{array}{c}\text { Cross-validation } \\
\text { accuracy }\end{array}$ & $100 \%$ & $100 \%$ & $65.85 \%$ & $82.98 \%$ & $100 \%$ \\
\hline & Mean CVA & \multicolumn{5}{|c|}{$89.77 \%$} \\
\hline
\end{tabular}

Figure 7. Number of features and cross-validation accuracy for each of the working conditions under consideration-(ES) engine speed, $(T O)$ threshing cylinder operation, $(T B)$ threshing cylinder balance, $(S C O)$ straw chopper operation, and $(S C B)$ straw chopper balance - using the accelerometer channel corresponding to (a) the transverse $X$ axis; (b) the longitudinal $Y$ axis; and (c) the vertical $Z$ axis.

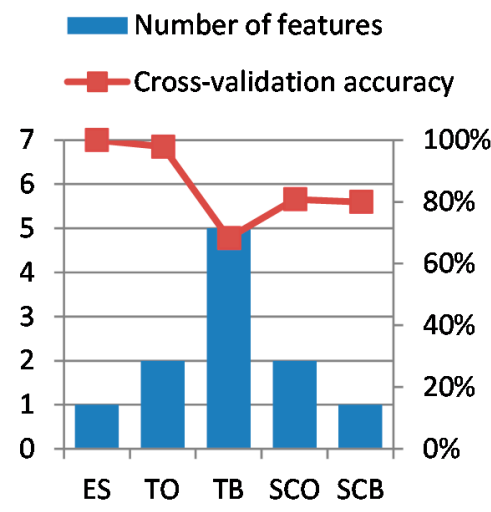

(a)

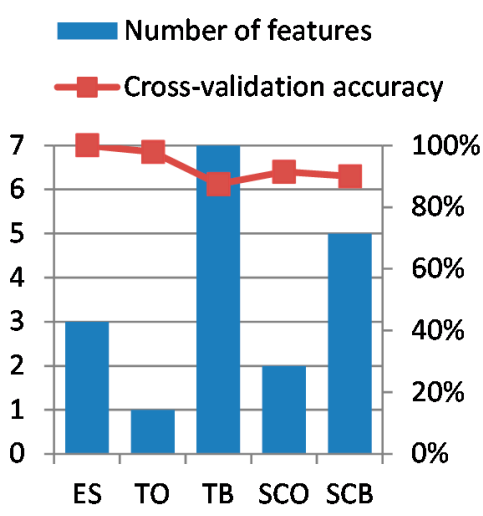

(b)

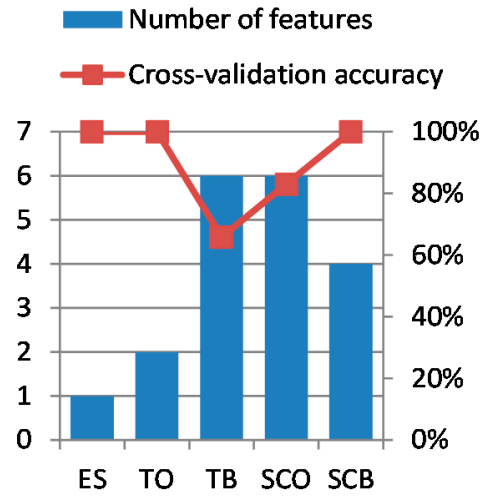

(c) 


\subsection{SVM Classifier Performance Evaluation}

The results of the linear and nonlinear SVM classifier optimization, showing the best cross-validation accuracies and the related optimal parameters, are shown in Table 2. The previously selected features, highlighted in bold in Table 1, were used as input to the SVM classifier. Note that the nonlinear kernels did not outperform the linear kernel in most cases. Even in those cases where the accuracy was improved, only slight differences never over 10\% were observed. It therefore appears that the use of linear SVM classification is sufficient to solve the problem. A comparison of kernel cross-validation accuracy is also provided in Figure 8.

Table 2. Performance results for each of the three axes acquired by the triaxial accelerometer, comparing the different SVM kernels, and showing both the optimized parameters $\left(C, \gamma, c_{0}\right)$ and the best cross-validation accuracy (CVA). The best result for each classification problem appears in bold.

\begin{tabular}{|c|c|c|c|c|c|c|c|}
\hline & \multicolumn{5}{|c|}{ Classification Problem } \\
\hline & & & $\begin{array}{l}\text { Engine } \\
\text { Speed }\end{array}$ & $\begin{array}{c}\text { Threshing } \\
\text { Cylinder Operation }\end{array}$ & $\begin{array}{c}\text { Threshing } \\
\text { Cylinder Balance }\end{array}$ & $\begin{array}{c}\text { Straw Chopper } \\
\text { Operation }\end{array}$ & $\begin{array}{c}\text { Straw Chopper } \\
\text { Balance }\end{array}$ \\
\hline \multirow{13}{*}{$\begin{array}{c}\text { Transverse } \\
X \text { axis of the } \\
\text { accelerometer }\end{array}$} & \multirow{2}{*}{ Linear kernel } & CVA & $100 \%$ & $97.87 \%$ & $75.61 \%$ & $80.85 \%$ & $90.00 \%$ \\
\hline & & $\mathrm{C}$ & 1 & 1 & 6 & 1 & 1.2 \\
\hline & \multirow{4}{*}{$\begin{array}{c}\text { Third-order } \\
\text { polynomial } \\
\text { kernel }\end{array}$} & CVA & $100 \%$ & $97.87 \%$ & $82.93 \%$ & $82.98 \%$ & $90.00 \%$ \\
\hline & & $\mathrm{C}$ & 0.03 & 32768 & 8192 & 2048 & 0.03 \\
\hline & & $\gamma$ & 8 & 0.125 & 0.125 & 32 & 8 \\
\hline & & $\mathrm{c}_{0}$ & 0.03 & 0.03 & 0.5 & 0.5 & 0.03 \\
\hline & \multirow{3}{*}{ RBF kernel } & CVA & $100 \%$ & $97.87 \%$ & $80.49 \%$ & $85.10 \%$ & $90.00 \%$ \\
\hline & & $\mathrm{C}$ & 0.125 & 512 & 32 & 32 & 0.5 \\
\hline & & $\gamma$ & 2 & 0.125 & 2 & 8 & 2 \\
\hline & \multirow{4}{*}{$\begin{array}{l}\text { Sigmoidal } \\
\text { kernel }\end{array}$} & CVA & $100 \%$ & $97.87 \%$ & $80.49 \%$ & $82.98 \%$ & $90.00 \%$ \\
\hline & & $\mathrm{C}$ & 2 & 2048 & 2048 & 8 & 2 \\
\hline & & $\gamma$ & 0.5 & 0.125 & 0.125 & 8 & 2 \\
\hline & & $\mathrm{c}_{0}$ & 0.03 & 0.03 & 0.03 & 0.5 & 0.03 \\
\hline \multirow{13}{*}{$\begin{array}{l}\text { Longitudinal } \\
Y \text { axis of the } \\
\text { accelerometer }\end{array}$} & \multirow{2}{*}{ Linear kernel } & CVA & $100 \%$ & $97.87 \%$ & $87.49 \%$ & $91.49 \%$ & $90.00 \%$ \\
\hline & & $\mathrm{C}$ & 1 & 1 & 1 & 1 & 1 \\
\hline & \multirow{4}{*}{$\begin{array}{c}\text { Third-order } \\
\text { polynomial } \\
\text { kernel }\end{array}$} & CVA & $100 \%$ & $97.87 \%$ & $80.49 \%$ & $91.49 \%$ & $90.00 \%$ \\
\hline & & $\mathrm{C}$ & 8192 & 0.03 & 8192 & 0.03 & 2048 \\
\hline & & $\gamma$ & 0.125 & 8 & 0.002 & 8 & 0.125 \\
\hline & & $\mathrm{c}_{0}$ & 0.03 & 0.03 & 8 & 0.03 & 0.03 \\
\hline & \multirow{3}{*}{ RBF kernel } & CVA & $100 \%$ & $97.87 \%$ & $78.05 \%$ & $91.49 \%$ & $90.00 \%$ \\
\hline & & $\mathrm{C}$ & 0.5 & 0.5 & 2 & 2 & 2 \\
\hline & & $\gamma$ & 8 & 8 & 0.5 & 2 & 2 \\
\hline & \multirow{4}{*}{$\begin{array}{l}\text { Sigmoidal } \\
\text { kernel }\end{array}$} & CVA & $100 \%$ & $97.87 \%$ & $78.05 \%$ & $93.62 \%$ & $90.00 \%$ \\
\hline & & $\mathrm{C}$ & 2 & 32 & 512 & 8192 & 8 \\
\hline & & $\gamma$ & 0.5 & 0.125 & 0.008 & 0.125 & 0.5 \\
\hline & & $\mathrm{c}_{0}$ & 0.03 & 0.03 & 0.03 & 0.125 & 0.03 \\
\hline \multirow{13}{*}{$\begin{array}{c}\text { Vertical } Z \text { axis } \\
\text { of the } \\
\text { accelerometer }\end{array}$} & \multirow{2}{*}{ Linear kernel } & CVA & $100 \%$ & $100 \%$ & $65.85 \%$ & $85.10 \%$ & $100 \%$ \\
\hline & & $\mathrm{C}$ & 1 & 1 & 1 & 430 & 1 \\
\hline & \multirow{4}{*}{$\begin{array}{l}\text { Third-order } \\
\text { polynomial } \\
\text { kernel }\end{array}$} & CVA & $100 \%$ & $100 \%$ & $63.41 \%$ & $89.36 \%$ & $100 \%$ \\
\hline & & $\mathrm{C}$ & 8192 & 512 & 2048 & 8192 & 0.03 \\
\hline & & $\gamma$ & 0.125 & 0.5 & 0.125 & 0.03 & 8 \\
\hline & & $\mathrm{c}_{0}$ & 0.03 & 0.03 & 0.5 & 2 & 0.03 \\
\hline & \multirow{3}{*}{ RBF kernel } & CVA & $100 \%$ & $100 \%$ & $68.29 \%$ & $87.23 \%$ & $100 \%$ \\
\hline & & $\mathrm{C}$ & 0.125 & 32 & 2 & 8192 & 2 \\
\hline & & $\gamma$ & 2 & 0.5 & 32 & 0.03 & 0.5 \\
\hline & \multirow{4}{*}{$\begin{array}{c}\text { Sigmoidal } \\
\text { kernel }\end{array}$} & CVA & $100 \%$ & $100 \%$ & $63.41 \%$ & $82.97 \%$ & $100 \%$ \\
\hline & & $\mathrm{C}$ & 2 & 128 & 512 & 32 & 8 \\
\hline & & $\gamma$ & 0.5 & 0.125 & 0.5 & 0.125 & 0.5 \\
\hline & & $\mathrm{c}_{0}$ & 0.03 & 0.03 & 0.125 & 0.03 & 0.03 \\
\hline
\end{tabular}


Figure 8. Cross-validation accuracy for each kernel under the following working conditions - $(E S)$ engine speed, $(T O)$ threshing cylinder operation, $(T B)$ threshing cylinder balance, $(S C O)$ straw chopper operation, and $(S C B)$ straw chopper balance-using the accelerometer channel corresponding to (a) the transverse $X$ axis; (b) the longitudinal $Y$ axis; and (c) the vertical $Z$ axis.

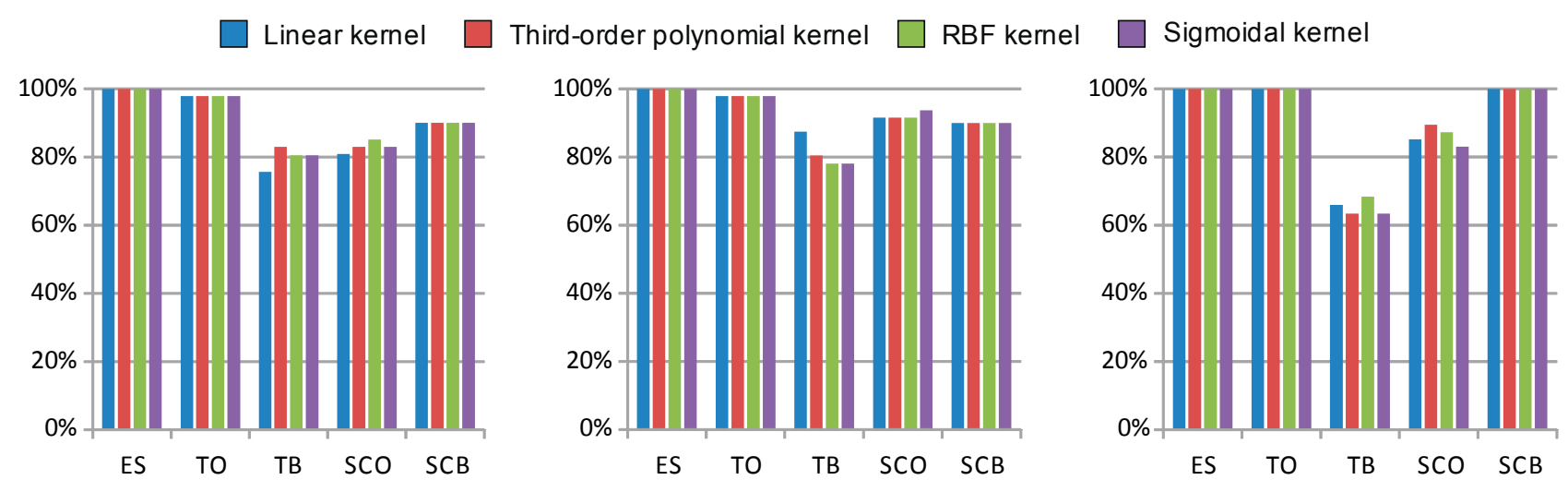

\section{Discussion}

This article investigates a method of estimating the status of various rotating components in agro-industrial machinery by processing vibration signals acquired from a single point of the machine structure. It offers three major findings.

The first finding of this article is that it is possible to accurately estimate the status of some rotating components in agro-industrial machinery by processing the vibration signal acquired from a single point on the machine. Moreover, the accelerometer sensor does not need to be placed very close to the rotating components, which makes the acquisition stage simple and non-intrusive. The results presented above reveal the potential of this method to estimate the status of distant components by processing vibration signals from a unique sensor located at a fixed position, midway along the harvester chassis (Figure 5), because a mean cross-validation accuracy higher than $85 \%$ was obtained. Previous work in the scientific literature has only analyzed isolated mechanical components, using one accelerometer for each isolated component $[19,21,56]$. It is worth noting that, to the best of our knowledge, no previous articles have approached the problem of estimating the status of various mechanical components from a unique vibration signal.

The second finding of this article is that the vibration signal can be acquired with a uniaxial accelerometer, the orientation of which has no significant effect on classification accuracy. The comparison of the results of cross-validation accuracy along the three accelerometer axes (Table 2) supports this conclusion. The higher differences observable in Table 2 for the threshing cylinder balance status shows differences of around 20\%. However, almost no differences in accuracy were appreciated for the rest of the states, which were lower than $10 \%$ in all cases. Although vibrations are usually generated in a specific direction, the results obtained here suggest that the machine structure spreads them along all of the axes, making the use of an arbitrary axis for their detection possible.

The third finding of this article is that, when using an SVM classifier, an 85\% mean cross-validation accuracy can be reached, which only requires a maximum of seven features as its input, with no 
significant noticeable improvements from using nonlinear rather than linear kernels. Reviewing the results, a mean cross-validation accuracy greater than $85 \%$ was achieved, irrespective of the selected accelerometer axis. Analyzing the individual cross-validation accuracy obtained for each rotating component, the suitability of the SVM classifier for estimating each separate machinery status is evident. On the one hand, the rotating component status with the best cross-validation accuracy was the engine speed, with a cross-validation accuracy of 100\% in all cases (Table 2). On the other hand, the worst cross-validation accuracy was obtained for threshing cylinder balance status, for which the cross-validation accuracy was between $63.41 \%$ and $87.49 \%$ (Table 2). A visual analysis of the vibration signal spectrum, revealed differences when the engine speed varied between high and low speed, while there were no visible changes in the signal spectrum when the threshing cylinder was either balanced or unbalanced. These results show that the proposed SVM classifier is able to classify the status of rotating machinery to a high degree of accuracy when the difference between the spectrum signals is noticeable, such as in the case of the engine speed status. They also show that it can obtain an acceptable cross-validation accuracy for rotating components when there is no visible difference between the spectrum signals, such as the threshing cylinder balance status. Comparing the fault detection accuracy in the present article against the results of Samanta et al. [56], who proposed an ANN-based classifier for the fault diagnostics of roller bearings based on data from several vibration signals and extracting only five time-domain features, this study has reported poorer results. Nevertheless, these differences can be justified by taking into account that in Samanta's article five vibration signals from different locations of a unique component were processed and because they were clean, as they came from the isolated mechanical component under analysis. Nevertheless, only one accelerometer sensor is employed in the present article to detect five states of three different rotating components and, furthermore, the vibration signal that is processed contains the superposed signals coming from the three components under analysis as well as from the other components of the machine. As can easily be understood, the present article approaches a much harder problem.

The major strength of the system proposed in this article is the simplicity of the data acquisition stage, employing only one sensor located at a single point on the machine for measuring the vibration signals. It is worth highlighting an article from Sugumaran et al. [21], who proposed an SVM-based classifier for the fault diagnostics of a unique roller bearing employing only one vibration signal. Our study, even though similar to Sugumaran's, is wider in the sense of trying to assess several machinery rotating components at once instead of just one. Furthermore, the present article contemplates the detection of further machine states and not only fault diagnostics.

Another strength of this article is that the proposed estimation method only needs seven features, at most, as the classifier input, yielding a simple SVM classifier with a low associated computational load. Moreover, the results showed no great differences in relation to the SVM kernel that was employed, which highlights that a simpler linear SVM classifier is sufficient to achieve good classification accuracy.

Nevertheless, there is a limitation to this work, which should be taken into account before implementing the proposed estimation method. This limitation is related to the data acquisition process performed in this article to validate the proposed SVM-based system. The vibration signals were acquired with the harvester wheels stopped to facilitate the acquisition procedure. If the proposed estimation method were to be used when the monitored machine is in motion, low-frequency 
interference signals could appear. However, these signals are not expected to cause problems, because the frequencies of interest in the rotating components of these machines will almost certainly be much higher than the interference frequencies.

The main application fields of the proposed SVM-based system are machinery monitoring and predictive maintenance. In relation to machinery monitoring, this system could be used for detecting the operating status of particular mechanical components, simplifying the wiring and reducing the number of sensors that are required. In relation to predictive maintenance, the results suggest that further progress may lead to fast and low-cost machinery inspections, thereby avoiding many mechanical faults and replacing expensive, time-consuming inspections that are frequently required nowadays.

A mixture of both conventional vibration signal analysis features, such as frequencydomain $[4,6,19,21,27,34]$ and time-domain [54-56] based features, and other unconventional features, such as nonlinear features $[33,37,39]$, has been used in this article. The good classification accuracy levels, obtained for example with the Central Tendency Measurement feature when estimating the engine speed status (Table 1), highlights the usefulness of these unconventional features in the analysis of vibration signals for predictive maintenance. Nevertheless, regarding the straw chopper unbalance detection, the nonlinear features seem to be of little use. A future line for further research is opened by employing other unconventional features in vibration analysis for predictive maintenance.

Furthermore, this article opens a new future line of research by extending the system that is proposed in this paper to the use of more than one accelerometer located at different points on the machine. It is expected that the processing of all those signals together could enable the estimation of an even higher number of machine states and could also improve the accuracy of the estimation.

\section{Conclusions}

The results obtained in this study have provided evidence that (i) accurate estimation of the status of various rotating components in agro-industrial machinery is possible by processing the vibration signal acquired from a single point on the machine structure; (ii) the vibration signal can be acquired with a uniaxial accelerometer, the orientation of which does not significantly affect the classification accuracy; and, (iii) when using an SVM classifier, an 85\% mean cross-validation accuracy can be reached, which only requires a maximum of seven features as its input, and no significant improvements are noted between the use of either nonlinear or linear kernels. Follow up research may lead to a simplification of the wiring and a reduction in the number of sensors required in machinery monitoring, as well as to fast and low cost machinery inspections in predictive maintenance.

\section{Acknowledgments}

Ruben Ruiz-Gonzalez was financed during this article's research by a collaboration grant from the Ministry of Education, Culture and Sport of Spain (BOE-A-2013-7736).

Víctor Martínez-Martínez's work was made possible thanks to a Formación de Personal Investigador program grant, financed by the Universidad de Valladolid (Spain) and co-financed by Banco Santander. 


\section{Author Contributions}

The work presented here was done in four stages. The first stage consisted in the work proposal and experiments design, and this stage was done collaboratively by the four authors. The second stage consisted in the realization of the experiments and the related data acquisition. This work was done in Aguilar de Bureba (Burgos, Spain) by Francisco Javier Gomez-Gil, Jaime Gomez-Gil, and Ruben Ruiz-Gonzalez. The third stage consisted in the data processing, and it was done by Ruben Ruiz-Gonzalez under the supervision of Jaime Gomez-Gil, Víctor Martínez-Martínez and Francisco Javier Gomez-Gil. The fourth stage was the article writing, which was done collaboratively by the four authors.

\section{Conflicts of Interest}

The authors declare no conflict of interest.

\section{References}

1. Martin, K.F. A review by discussion of condition monitoring and fault diagnosis in machine tools. Int. J. Mach. Tools Manuf. 1994, 34, 527-551.

2. Jardine, A.K.S.; Lin, D.; Banjevic, D. A review on machinery diagnostics and prognostics implementing condition-based maintenance. Mech. Syst. Signal Proc. 2006, 20, 1483-1510.

3. Other predictive maintenance techniques. In Practical Machinery Vibration Analysis and Predictive Maintenance; Girdhar, P., Scheffer, C., Eds.; Newnes: Oxford, UK, 2004; pp. 221-234.

4. Scheffer, C.; Girdhar, P. Practical Machinery Vibration Analysis and Predictive Maintenance; Newnes: Oxford, UK, 2004.

5. Wang, W.Q.; Ismail, F.; Farid Golnaraghi, M. Assessment of gear damage monitoring techniques using vibration measurements. Mech. Syst. Signal Proc. 2001, 15, 905-922.

6. Peng, Z.K.; Chu, F.L. Application of the wavelet transform in machine condition monitoring and fault diagnostics: A review with bibliography. Mech. Syst. Signal Proc. 2004, 18, 199-221.

7. Li, Z.; Yan, X.; Tian, Z.; Yuan, C.; Peng, Z.; Li, L. Blind vibration component separation and nonlinear feature extraction applied to the nonstationary vibration signals for the gearbox multi-fault diagnosis. Measurement 2013, 46, 259-271.

8. Albarbar, A.; Gu, F.; Ball, A.D. Diesel engine fuel injection monitoring using acoustic measurements and independent component analysis. Measurement 2010, 43, 1376-1386.

9. Taghizadeh-Alisaraei, A.; Ghobadian, B.; Tavakoli-Hashjin, T.; Mohtasebi, S.S. Vibration analysis of a diesel engine using biodiesel and petrodiesel fuel blends. Fuel 2012, 102, 414-422.

10. Vulli, S.; Dunne, J.F.; Potenza, R.; Richardson, D.; King, P. Time-frequency analysis of single-point engine-block vibration measurements for multiple excitation-event identification. J. Sound Vibr. 2009, 321, 1129-1143.

11. Wang, X.; Makis, V.; Yang, M. A wavelet approach to fault diagnosis of a gearbox under varying load conditions. J. Sound Vibr. 2010, 329, 1570-1585. 
12. Bin, G.F.; Gao, J.J.; Li, X.J.; Dhillon, B.S. Early fault diagnosis of rotating machinery based on wavelet packets-Empirical mode decomposition feature extraction and neural network. Mech. Syst. Signal Proc. 2012, 27, 696-711.

13. Chen, F.; Tang, B.; Chen, R. A novel fault diagnosis model for gearbox based on wavelet support vector machine with immune genetic algorithm. Measurement 2013, 46, 220-232.

14. Rodriguez-Donate, C.; Romero-Troncoso, R.; Cabal-Yepez, E.; Garcia-Perez, A.; Osornio-Rios, R. Wavelet-based general methodology for multiple fault detection on induction motors at the startup vibration transient. J. Vib. Control 2011, 17, 1299-1309.

15. Jayaswal, P.; Verma, S.; Wadhwani, A. Development of EBP-Artificial neural network expert system for rolling element bearing fault diagnosis. J. Vib. Control 2011, 17, 1131-1148.

16. McFadden, P.D.; Cook, J.G.; Forster, L.M. Decomposition of gear vibration signals by the Generalised S Transform. Mech. Syst. Signal Proc. 1999, 13, 691-707.

17. Wang, Y.S.; Ma, Q.H.; Zhu, Q.; Liu, X.T.; Zhao, L.H. An intelligent approach for engine fault diagnosis based on Hilbert-Huang transform and support vector machine. Appl. Acoust. 2014, 75, 1-9.

18. Lei, Y.; Lin, J.; He, Z.; Zuo, M.J. A review on empirical mode decomposition in fault diagnosis of rotating machinery. Mech. Syst. Signal Proc. 2013, 35, 108-126.

19. Cheng, G.; Cheng, Y.-L.; Shen, L.-H.; Qiu, J.-B.; Zhang, S. Gear fault identification based on Hilbert-Huang transform and SOM neural network. Measurement 2013, 46, 1137-1146.

20. Li, Y.; Tse, P.W.; Yang, X.; Yang, J. EMD-based fault diagnosis for abnormal clearance between contacting components in a diesel engine. Mech. Syst. Signal Proc. 2010, 24, 193-210.

21. Sugumaran, V.; Ramachandran, K.I. Effect of number of features on classification of roller bearing faults using SVM and PSVM. Expert Syst. Appl. 2011, 38, 4088-4096.

22. Vapnik, V. N. Statistical Learning Theory; Wiley: New York, NY, USA, 1998; p. 736.

23. Moguerza, J.M.; Muñoz, A. Support vector machines with applications. Statist. Sci. 2006, 21, 322-336.

24. Joachims, T. Learning to Classify Text Using Support Vector Machines: Methods, Theory and Algorithms; Kluwer Academic Publishers: New York, NY, USA, 2002; p. 205.

25. Wu, C.-H.; Ho, J.-M.; Lee, D.T. Travel-time prediction with support vector regression. IEEE Trans. Intell. Transp. Syst. 2004, 5, 276-281.

26. Su, H.; Shi, T.; Chen, F.; Huang, S. New method of fault diagnosis of rotating machinery based on distance of information entropy. Front. Mech. Eng. 2011, 6, 249-253.

27. Dai, J.; Chen, C.L.P.; Xu, X.-Y.; Huang, Y.; Hu, P.; Hu, C.-P.; Wu, T. Machinery vibration signals analysis and monitoring for fault diagnosis and process control. In Advanced Intelligent Computing Theories and Applications. With Aspects of Theoretical and Methodological Issues; Huang, D.-S., Wunsch, D., II, Levine, D., Jo, K.-H., Eds.; Springer: Berlin, Germany, 2008; Volume 5226, pp. 696-703.

28. Widodo, A.; Yang, B.-S. Support vector machine in machine condition monitoring and fault diagnosis. Mech. Syst. Signal Proc. 2007, 21, 2560-2574.

29. Jain, A.K.; Duin, R.P.W.; Mao, J. Statistical pattern recognition: A review. IEEE Trans. Pattern Anal. Mach. Intell. 2000, 22, 4-37. 
30. Kotsiantis, S.B. Supervised machine learning: A review of classification techniques. Informatica 2007, 31, 249-268.

31. Doğanaksoy, A.; Göloğlu, F. On Lempel-Ziv Complexity of Sequences. In Sequences and Their Applications—SETA 2006; Gong, G., Helleseth, T., Song, H.-Y., Yang, K., Eds.; Springer: Berlin, Germany, 2006; Volume 4086, pp. 180-189.

32. Vanluchene, A.L.; Vereecke, H.; Thas, O.; Mortier, E.P.; Shafer, S.L.; Struys, M.M. Spectral entropy as an electroencephalographic measure of anesthetic drug effect: A comparison with bispectral index and processed midlatency auditory evoked response. Anesthesiology 2004, $1,34-42$.

33. Hornero, R.; Escudero, J.; Fernandez, A.; Poza, J.; Gomez, C. Spectral and nonlinear analyses of MEG background activity in patients with Alzheimer's disease. IEEE Trans. Biomed. Eng. 2008, $55,1658-1665$.

34. Gao, G.; Zhu, Y.; Duan, G.; Zhang, Y. Intelligent fault identification based on wavelet packet energy analysis and SVM. In Proceedings of the 9th International Conference on Control, Automation, Robotics and Vision (ICARCV'06), Singapore, 5-8 December 2006; pp. 1-5.

35. Grassberger, P.; Procaccia, I. Measuring the strangeness of strange attractors. Phys. D 1983, 9, 189-208.

36. Li, M.; Vitányi, P. An Introduction to Kolmogorov Complexity and Its Applications, 3rd ed.; Springer: New York, NY, USA, 2008; p. 792.

37. Aboy, M.; Hornero, R.; Abásolo, D.; Álvarez, D. Interpretation of the Lempel-Ziv complexity measure in the context of biomedical signal analysis. IEEE Trans. Biomed. Eng. 2006, 53, 2282-2288.

38. Pincus, S.M. Approximate entropy as a measure of system complexity. Proc. Natl. Acad. Sci. USA 1991, 88, 2297-2301.

39. Richman, J.S.; Moorman, J.R. Physiological time-series analysis using approximate entropy and sample entropy. Am. J. Physiol. Heart Circ. Physiol. 2000, 278, H2039-H2049.

40. Aggarwal, N.; Agrawal, R.K. First and Second order statistics features for classification of magnetic resonance brain images. J. Signal Inf. Proc. 2012, 3, 146-153.

41. Zhou, B.-H.; Liu, P.; Liu, N.B.; Wu, X.H.; Lu, R.; Ni, X.M. Median frequency of surface EMG signal of antagonist muscles during repeated contractions. In Proceeding of the 1992 14th Annual International Conference of the IEEE Engineering in Medicine and Biology Society, Paris, France, 29 October-1 November 1992; Volume 4, pp. 1626-1627.

42. Wang, X.; Paliwal, K.K. Feature extraction and dimensionality reduction algorithms and their applications in vowel recognition. Pattern Recognit. 2003, 36, 2429-2439.

43. Cortes, C.; Vapnik, V. Support-Vector networks. Mach. Learn. 1995, 20, 273-297.

44. Haykin, S. Neural Networks: A Comprehensive Foundation, 2nd ed.; Prentice Hall PTR: New Jersey, NJ, USA, 1998; p. 842.

45. Ren, J. ANN vs. SVM: Which one performs better in classification of MCCs in mammogram imaging. Knowl. Based Syst. 2012, 26, 144-153.

46. Hsu, C.-W.; Lin, C.-J. A comparison of methods for multiclass support vector machines. IEEE Trans. Neural Netw. 2002, 13, 415-425. 
47. Ferris, M.C.; Munson, T.S. Interior-point methods for massive support vector machines. SIAM J. Optim. 2006, 13, 783-804.

48. Platt, J.C. Sequential Minimal Optimization: A Fast Algorithm for Training Support Vector Machines; Microsoft Research Technical Report MSR-TR-98-14, 21 April 1998.

49. Platt, J.C. Fast training of support vector machines using sequential minimal optimization. In Advances in Kernel Methods: Support Vector Learning, 1st ed.; Schölkopf, B., Burges, C.J.C., Smola, A.J., Eds.; MIT Press: Cambridge, UK, 1998.

50. Shilton, A.; Palaniswami, M.; Ralph, D.; Tsoi, A.C. Incremental training of support vector machines. IEEE Trans. Neural Netw. 2005, 16, 114-131.

51. Campbell, C.; Cristianini, N. Simple Learning Algorithms for Training Support Vector Machines; University of Bristol: Bristol, UK, 1998.

52. Campbell, C.; Ying, Y. Learning with Support Vector Machines, 1st ed.; Morgan \& Claypool Publishers: San Rafael, CA, USA, 2011.

53. Steinwart, I.; Christmann, A. Support Vector Machines, 1st ed.; Springer Publishing Company, Inc.: New York, NY, USA, 2008.

54. Norton, M.P.; Karczub, D.G. Fundamentals of Noise and Vibration Analysis for Engineers; Cambridge University Press: Cambridge, UK, 2003.

55. Tandon, N. A comparison of some vibration parameters for the condition monitoring of rolling element bearings. Measurement 1994, 12, 285-289.

56. Samanta, B.; Al-Balushi, K.R. Artificial Neural Network based fault diagnostics of rolling element bearings using time-domain features. Mech. Syst. Signal Proc. 2003, 17, 317-328.

57. Chang, C.-C.; Lin, C.-J. LIBSVM: A library for support vector machines. ACM Trans. Intell. Syst. Technol. 2011, 2, 27:1-27:27.

58. Chang, C.-C.; Lin, C.-J. LIBSVM-A Library for Support Vector Machines. Available online: http://www.csie.ntu.edu.tw/ cjlin/libsvm (accessed on 3 August 2014).

59. Hsu, C.-W.; Chang, C.-C.; Lin, C.-J. A Practical Guide to Support Vector Classification; Department of Computer Science and Information Engineering, National Taiwan University: Taibei, Taiwan, 2010.

(C) 2014 by the authors; licensee MDPI, Basel, Switzerland. This article is an open access article distributed under the terms and conditions of the Creative Commons Attribution license (http://creativecommons.org/licenses/by/4.0/). 